\title{
ON THE SERIAL RELATION IN BOOLEAN ALGEBRAS*
}

BY B. A. BERNSTEIN

The elements $x, y, z, \cdots$ of a class $K$ are said to form a series with respect to a dyadic relation $S$, if they satisfy the following postulates $\dagger$

$$
\begin{array}{ll}
P_{1} . & \text { If } x \neq y, \text { then either } x S y \text { or } y S x . \\
P_{2} . & \text { If } x S y, \text { then } x \neq y . \\
P_{3} . & \text { If } x S y \text { and } y S z \text {, then } x S z .
\end{array}
$$

It is my object to determine all serial relations in Boolean algebras given by universal propositions expressible in the fundamental Boolean operations of addition, multiplication, and negation.

All the desired serial relations $S$ must be of the form

$$
a x y+b x y^{\prime}+c x^{\prime} y+d x^{\prime} y^{\prime}=0
$$

where $x^{\prime}$ is the negative of $x$. Our problem then reduces itself to finding the conditions imposed on the coefficients of (1) by $P_{1}-P_{3}$. We proceed to determine these conditions.

If in (1) $x=0$ and $y=1$, then $c=0$; if $x=1$ and $y=0$, then $b=0$. Hence the condition imposed on (1) by $P_{1}$ is

$$
b=0 \quad \text { or } \quad c=0 .
$$

The condition imposed on (1) by $P_{2}$ is that the equation $a x x+b x x^{\prime}+c x^{\prime} x+d x^{\prime} x^{\prime}=0$ have no solution. This condition is

$$
a d \neq 0 .
$$

The condition that (1) satisfies $P_{3}$ non-vacuously is (this Bulletin, vol. 30, p. 127)

$$
a+d<b+c, a d=0 \text {, }
$$

which contradicts (3). Hence, there are no non-vacuous serial relations (1) in any Boolean algebra.

* Presented to the Society, San Francisco Section, June 12, 1926.

$\dagger$ See E. V. Huntington, The Continuum, 2d ed., p. 10. 
But there may possibly be vacuous serial relations (1). To determine these, let us first find the condition that there be no $x, y, z$ such that both $x S y$ and $y S z$ hold, i. e., the condition that there be no $x, y, z$ such that simultaneously

$$
\begin{aligned}
& a x y+b x y^{\prime}+c x^{\prime} y+d x^{\prime} y^{\prime}=0, \\
& a y z+b y z^{\prime}+c y^{\prime} z+d y^{\prime} z^{\prime}=0 .
\end{aligned}
$$

Since (i) and (ii) together are equivalent to the single equation

(iii) $a x y z+(b+c) x y^{\prime} z+(b+c) x^{\prime} y z+(c+d) x^{\prime} y^{\prime} z$

$$
+(a+b) x y z^{\prime}+(b+d) x y^{\prime} z^{\prime}+(b+c) x^{\prime} y z^{\prime}+d x^{\prime} y^{\prime} z^{\prime}=0,
$$

our condition is found to be

$$
a d(b+c) \neq 0 .
$$

Now $a$ in (1) must be either 0 or 1 , for otherwise neither $1 S a$ nor $a S 1$, contrary to $P_{1}$. Similarly $b, c, d$ must be either 0 or 1 . Hence, taking account of (2) and (5), we find that all vacuous serial relations (1) must be among the relations

$$
x y+x y^{\prime}+x^{\prime} y^{\prime}=0, \quad x y+x^{\prime} y+x^{\prime} y^{\prime}=0 .
$$

Since for $e \neq 0,1$ neither equation of (6) is satisfied by $x=1$ and $y=e$, or by $x=e$ and $y=1$, we see that there are no vacuous serial relations (1) in a Boolean algebra consisting of more than two elements.

However, equations (6) are serial relations in a twoelement Boolean algebra, since they produce respectively the relation-tables (this BulLETIN vol, 30, p. 27):

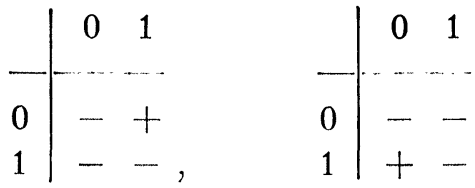

We may sum up our results as follows: Relations (6) are the only serial relations of form (1) in Boolean algebras; both of these relations are vacuous and both are confined to the Boolean algebra of order two.

The University of California 